\title{
Adult Perceptions of Positive and Negative Infant Emotional Expressions
}

\author{
Laura H. Bolzani Dinehart, Daniel S. Messinger, \\ Susan I. Acosta, and Tricia Cassel \\ Department of Psychology \\ University of Miami
}

\author{
Zara Ambadar and Jeffrey Cohn \\ Department of Psychology \\ University of Pittsburgh
}

\begin{abstract}
Adults' perceptions provide information about the emotional meaning of infant facial expressions. This study asks whether similar facial movements influence adult perceptions of emotional intensity in both infant positive (smile) and negative (cry face) facial expressions. Ninety-five college students rated a series of naturally occurring and digitally edited images of infant facial expressions. Naturally occurring smiles and cry faces involving the co-occurrence of greater lip movement, mouth opening, and eye constriction, were rated as expressing stronger positive and negative emotion, respectively, than expressions without these 3 features. Ratings of digitally edited expressions indicated that eye constriction contributed to higher ratings of positive emotion in smiles (i.e., in Duchenne smiles) and greater eye constriction contributed to higher ratings of negative emotion in cry faces. Stronger mouth opening contributed to higher ratings of arousal in both smiles and cry faces. These findings indicate a set of similar facial movements are linked to perceptions of greater emotional intensity, whether the movements occur in positive or negative infant emotional expressions. This proposal is discussed with reference to discrete, componential, and dynamic systems theories of emotion.
\end{abstract}

Early positive emotional expressivity is associated with secure attachment (Cohn, Campbell, \& Ross, 1991) and greater sociality (Fox, Henderson, Rubin, Calkins,

Supplementary materials to this article are available on the World Wide Web at http://www.infancyarchives.com

Correspondence should be addressed to Daniel S. Messinger or Laura H. Bolzani Dinehart, Department of Psychology, University of Miami, P.O. Box 249229, Coral Gables, FL 33124-0721. E-mail: dmessinger@miami.edu or lbolzani@miami.edu 
\& Schmidt, 2001; Kagan, 1997), whereas negative emotional expressivity is associated with internalizing behaviors (Moore, Cohn, \& Campbell, 2001) and a propensity toward inhibition (Fox et al., 2001; Kagan, 1997). Yet smiles can communicate a range of positive emotional intensity and infant cry faces can communicate a range of negative emotional intensity (Messinger, 2002; Oster, 2000). This study examines the impact that specific facial features have on adult perceptions of emotional intensity in smiles and in cry faces. Identification of these facial features would increase our understanding of the types of emotion expression especially important to interaction and development. If similar facial features were involved in more emotionally intense smiles and cry faces, this would suggest a noteworthy economy of expression in infant emotional communication.

\section{POSITIVE AND NEGATIVE EXPRESSIONS}

In infants, positive emotions are generally expressed by a smile (Messinger, Fogel, \& Dickson, 2001; Sroufe, 1979) in which zygomatic major pulls the lip corners obliquely toward the cheekbones (Facial Action Coding System [FACS], AU 12; Ekman \& Friesen, 1978). In smiles with eye constriction (cheek raise or Duchenne smiles), obicularis oculi, pars lateralis (AU6) raises the cheeks toward the eyes and tightens the skin lateral to the eyes (producing crow's feet in adults; Oster, 2000). In adults and infants, smiles involving eye constriction occur during circumstances (Ekman, Davidson, \& Friesen, 1990), such as mother's smiling approach (Fox \& Davidson, 1988), indicative of positive affect. Between 1 and 6 months, smiling with eye constriction and with mouth opening are both more likely during positive periods of naturalistic interaction (Messinger et al., 2001). Infant smiling involving both eye constriction and mouth opening, for example, occurred while the infant was gazing at his or her smiling mother. Lip corner movement appears to be a linked index of emotional intensity (Oster, 2000, 2005; Segal et al., 1995) that is greatest in smiles involving both mouth opening and eye constriction, and is less strong when the smile involves only one of these features (Fogel, Hsu, Shapiro, Nelson-Goens, \& Secrist, in press). In sum, greater smiling lip corner movement, mouth opening, and eye constriction appear to be involved in the expression of intense positive emotion.

Cry faces are the prototypic infant response to negative stimuli (Camras, 1992). Cry faces encompass both discrete anger and distress or pain expressions (Izard, 1979). These two expressions often occur in response to the same negative events, and are both perceived as high in distress (Bennett, Bendersky, \& Lewis, 2002; Camras, Oster, Campos, Miyake, \& Bradshaw, 1992; Oster, Hegley, \& Nagel, 1992). In cry faces, the lips are stretched laterally by risorius (AU20) and the brows are lowered by the corrugator (AU4). Cry faces typically involve raising of the upper lip (AU10) and might involve nose wrinkling (AU9). Cry faces typically in- 
volve at least a minimal degree of mouth opening (jaw drop) and eye constriction (Camras et al., 1992; Oster, 2000; Segal et al., 1995).

Factors similar but not identical to those involved in more emotionally positive smiling - stronger lip movement, eye constriction, and mouth opening-also appear to characterize more emotionally negative cry faces. BabyFACS (Oster, 2000) suggests that the intensity of the cry face configuration varies with the degree of horizontal stretching of the lip corners and the degree of mouth opening involved. Stronger cry face lip corner movement also appears to be associated with greater eye constriction (Oster, 2000). Cry face expressions involving greater mouth opening (indexed by crying) are associated with patterns of cerebral activation (Fox \& Davidson, 1988) indicative of a predilection toward negative affect (Davidson \& Fox, 1989).

Despite these parallels, there are differences between smiles and cry faces in lip movement, eye constriction, and mouth opening. Smiles involve oblique pulling of the lip corners, whereas cry faces involve lateral lip stretching. Orbicularis oculi, pars lateralis (cheek raising) produces eye constriction in both smiles and cry faces; but orbicularis oculi, pars palpebralis, which tightens the eyelids, is also typically active in cry faces, but not smiles. Although the source of mouth opening is similar in smiles and cry faces, cry faces typically involve some mouth opening that combines with lip stretching to produce a characteristic square mouth formation, whereas smiles occur with both closed and open mouth.

\section{PREVIOUS RATING STUDIES OF INFANT EXPRESSIONS}

Adult ratings of emotion terms can provide insight into the meaning of infant facial expressions (Oster, 2003). Feeling typically refers to a subjective experience, whereas emotion refers to a broader, coordinated experiential, physiological, and expressive process. Emotional intensity refers to strength of subjective feeling and clarity of affective communication. Intensity is operationalized here as higher ratings of positive emotion and negative emotion (which are operationalized as composite of ratings of different emotion words) and higher ratings of arousal (see later).

Messinger (2002) found that participants rated a single infant's smiles with eye constriction as expressing more positive emotion than smiles without eye constriction. Participants rated cry faces with greater eye constriction made by the same infant as expressing stronger negative emotion than cry faces with weaker eye constriction. Eye constriction had large effects (accounting for $40 \%$ to $60 \%$ of effect-related variance) on raters' perceptions of the intensity of positive and negative emotion. Participants also rated smiles and cry faces with stronger lip movement and mouth opening as displaying stronger emotion than smiles and cry faces 
without. It was not possible to determine, however, whether strength of lip movement and presence of mouth opening had an independent effect on adults' perceptions of infant emotion.

The facial movements that constitute emotional expressions may communicate not only the intensity of positive and negative emotion but specific emotional dimensions such as arousal (Smith \& Scott, 1997). Arousal refers to an emotion-relevant state involving increased autonomic and physical activity. Although arousal can be expressed by the overall intensity of an expression (Frijida, Kuipers, \& Schure, 1989; Smith \& Scott, 1997), it can be especially indexed by mouth opening (Bolzani, Messinger, Yale, \& Dondi, 2002; Messinger, 2002; Russell, 1997). Infant open mouth smiles, for example, tend to occur when the infant is gazing at mother, a socially arousing action. In nonhuman primates mouth opening expressions occur during rough-and-tumble play that might also be associated with arousal (Redican, 1975). In cry faces, mouth opening appears to be linked to the arousal associated with vocal crying (Messinger, 2002). If mouth opening is associated with arousal, then expressions involving greater mouth opening should be perceived as involving more arousal than expressions involving less mouth opening.

This study examined perceptions of positive and negative emotional intensity and arousal, using both naturally occurring and digitally edited images of infant facial expressions. We first asked whether adults rated naturally occurring smiles and cry faces involving the co-occurrence of greater lip movement, mouth opening, and eye constriction as expressing stronger emotion than expressions without those three features. We next digitally edited smiles and cry faces to produce expressions that differed only with respect to lip movement, mouth opening, or eye constriction. Precise, computer vision measurements of lip corner movement and mouth opening informed the selection of pairs of expressions amenable to digital editing of these features (Cohn \& Kanade, in press; Xiao, Kanade, \& Cohn, 2003). Having digitally edited expressions that differed only in selected facial movements (greater lip movement, mouth opening, or eye constriction), we asked whether smiles and cry faces involving each of these movements were rated as expressing greater positive emotion, negative emotion, and arousal than expressions with lesser degrees of these movements.

\section{METHOD}

\section{Participants}

One hundred University of Miami undergraduates rated 37 infant facial expressions. The students received extra credit from their introductory psychology classes for their participation. Four participants were excluded from the analyses 
because they reported English was not their best language and 1 because she had already participated in a similar study. The remaining 95 participants were predominantly female $(62.5 \%)$, with a mean age of 19.7 years $(S D=3.3)$, and identified themselves as White (55\%), Hispanic (14\%), African American (15\%), Asian $(11 \%)$, and biracial, unidentified, or other $(5 \%)$.

\section{Capturing and Measuring Expressions}

To capture and create expressions, 4 infants were digitally videotaped participating in a face-to-face/still-face procedure with their mothers (Tronick, Als, Adamson, Wise, \& Brazelton, 1978). Short sequences of smiles, cry faces, or both from each infant were then selected based on the clarity of the videotaped image and the presence of the various facial features of interest. Within sequences, individual images (frames) were exported that constituted the naturally occurring expressions rated in the study and were the basis of the digitally edited expressions. Eight smiles came from Infant 1 (female; 8 months, 17 days), eight from Infant 2 (female; 6 months, 0 days), and two from Infant 4 (female; 9 months, 13 days; see Table 1). Eight cry faces came from Infant 1, eight from Infant 3 (male; 8 months, 16 days), and three from Infant 4 (see Table 2).

Three FACS-certified coders trained in BabyFACS (Oster, 2000) coded all expressions. Their codes were combined into a consensus code for each expression. Lip corner movement and mouth opening in the Infant 1 smiles and cry faces and the Infant 2 smiles were also measured with the Feature Point Tracking module of the CMU/Pitt automated facial image analysis (AFA-3) system (Cohn \& Kanade, in press; Cohn et al., 2004; Xiao et al., 2003). In AFA-3, head motion is recovered automatically and used to warp (or stabilize) the face to a standard view. In this way, motion of facial features is not confounded with head motion or variation in camera orientation or pose. Using AFA-3, mouth opening was measured as the distance between the top and bottom lips. Lip corner movement was measured as the average distance between each lip corner and the point directly between the lip corners in the first frame of the digitized sequence. Excessive head movement prevented measurement of the facial movement of the Infant 3 cry faces and the Infant 4 smiles and cry faces.

\section{Naturally Occurring Expressions}

Eight naturally occurring expressions - two pairs of smiles and two pairs of cry faces-involving co-occurrences of facial movements that might index emotional intensity were chosen for rating (see Table 3 ). Pairs of smiles and cry faces were selected from Infants 1, 2, and 3 that involved (a) an expression that involved weak lip movement, minimal mouth opening, and minimal to no eye constriction; and (b) an expression that involved stronger lip movement, and a greater degree of 


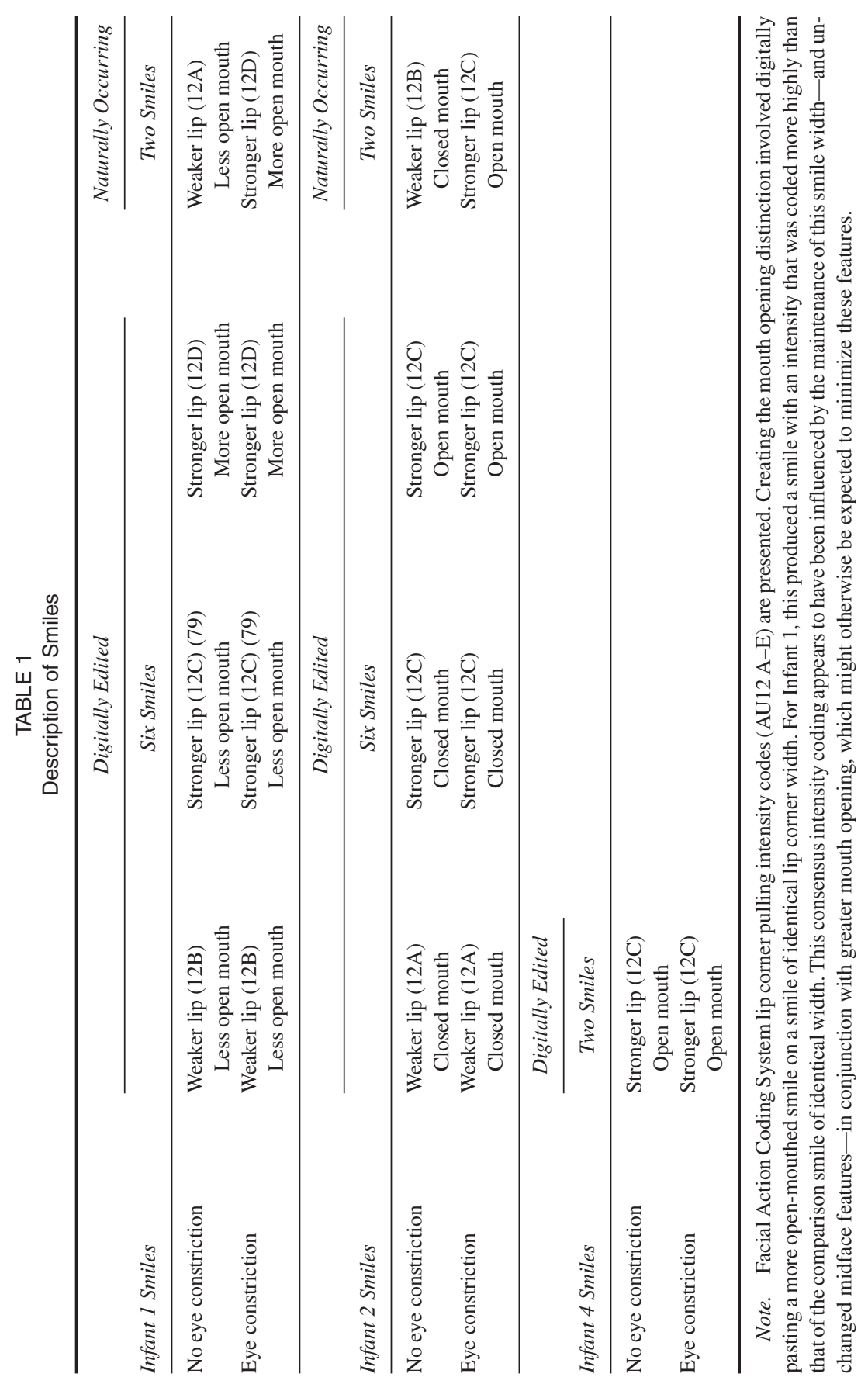




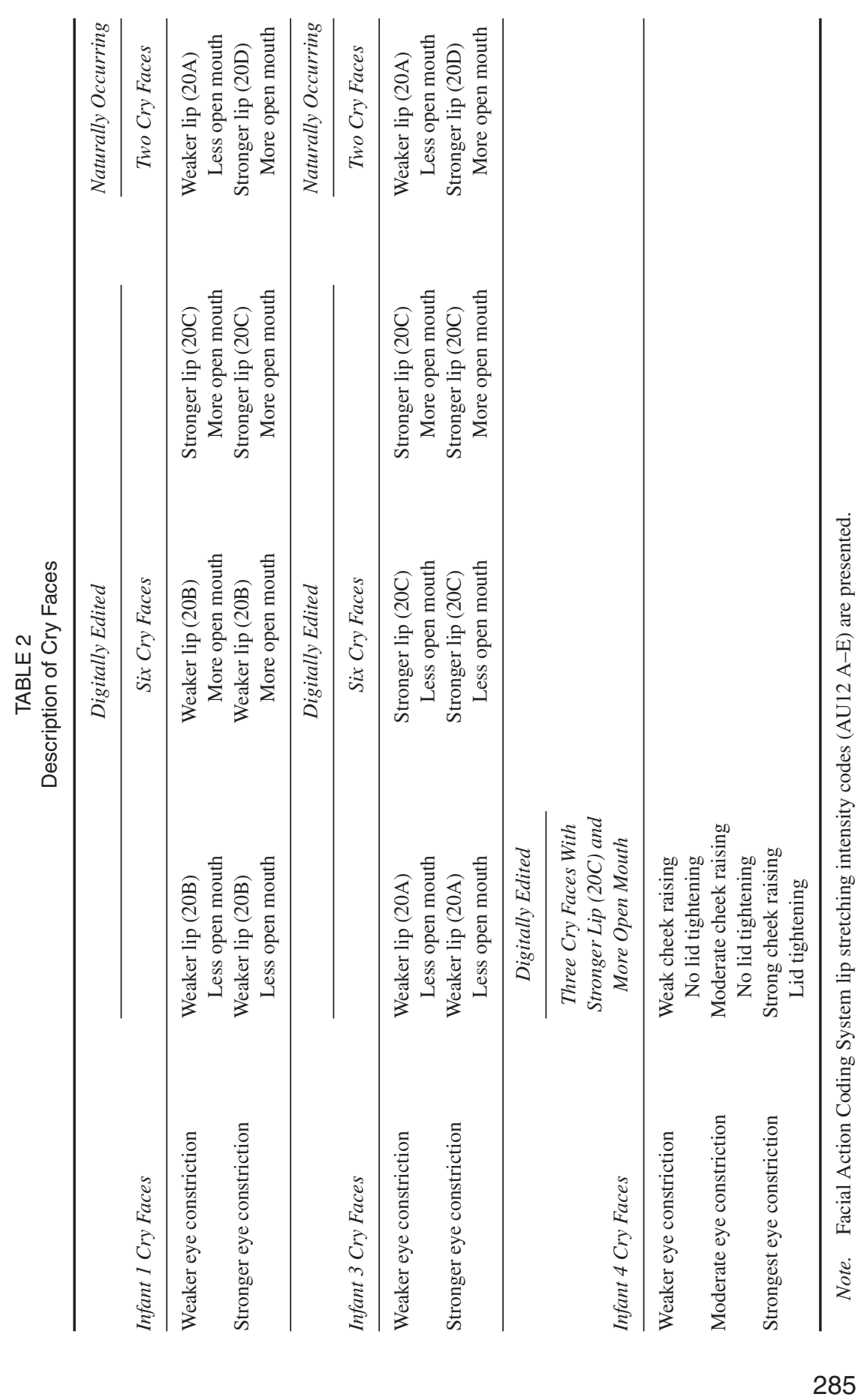




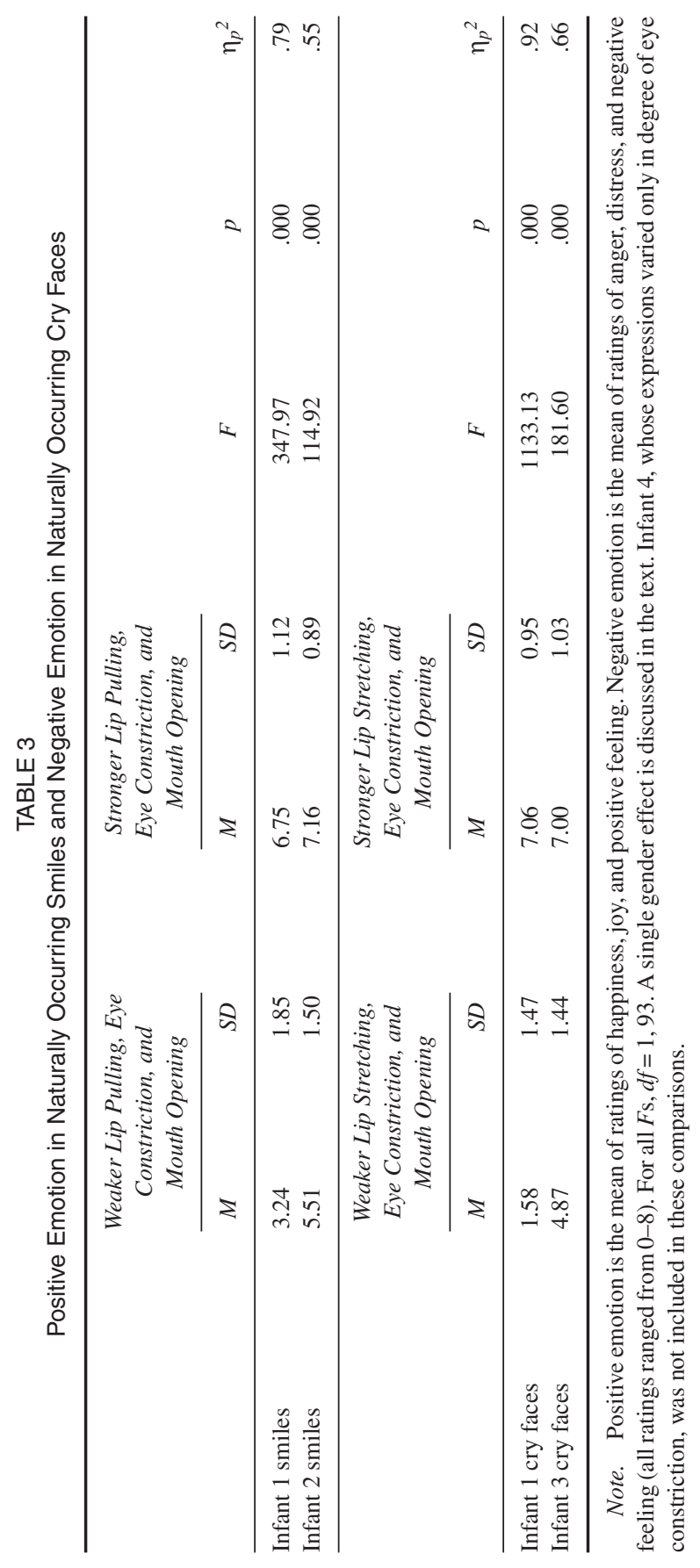


mouth opening and eye constriction. The goal was to determine whether naturally occurring smiles and cry faces involving this ensemble of features were rated higher, respectively, on positive emotion and negative emotion than expressions without this ensemble of features.

\section{Digitally Edited Expressions}

Digital editing of facial expressions using Adobe Photoshop was used to create sets of six digitally edited expressions for each infant that systematically contrasted lip movement, mouth opening, and eye constriction (see Tables $1 \& 2$ ). For each set of six, half the expressions involved greater eye constriction and half involved either no eye constriction (smiles) or weaker eye constriction (cry faces; see Figure 1). In each set, two weaker and two stronger lip movement expressions contrasted degree of lip movement while mouth opening was held constant. Finally, in each set, two expressions with greater mouth opening and two expressions with no or minimal mouth opening contrasted degree of mouth opening while strength of lip movement was held constant (see Figure 2).

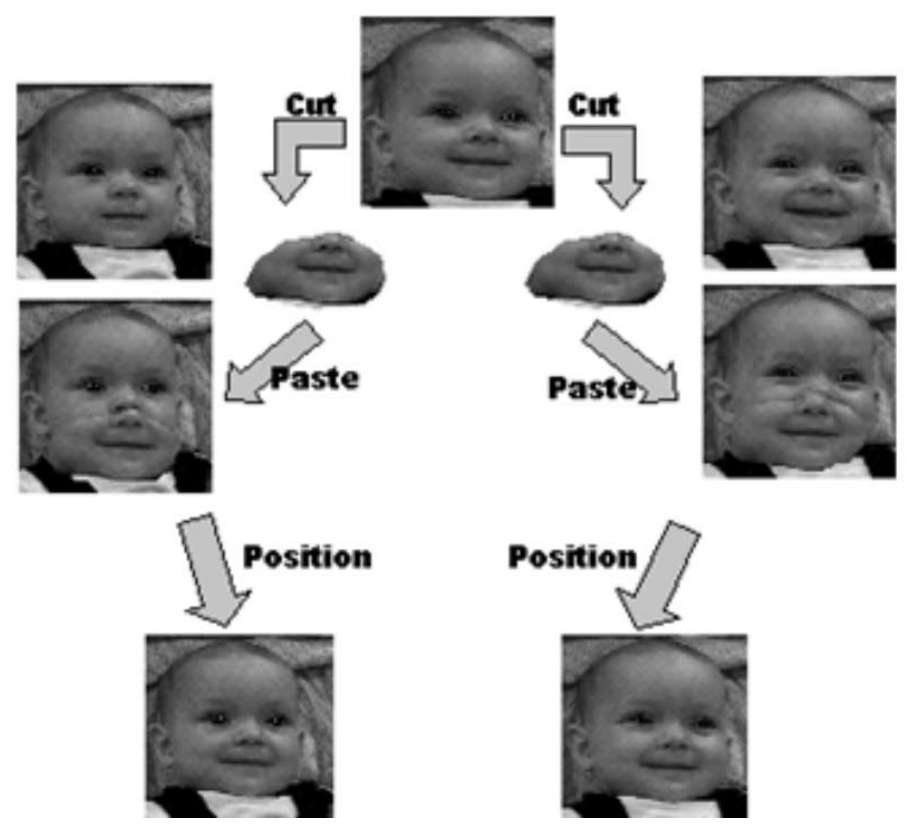

FIGURE 1 Creating eye constriction comparisons. Identical portions of the lower portion of an expression (from the cheekbones down) were cut, pasted, and positioned over one expression with eye constriction and one expression with minimal or no eye constriction. This allowed for the comparison of ratings of expressions varying only in eye constriction. 


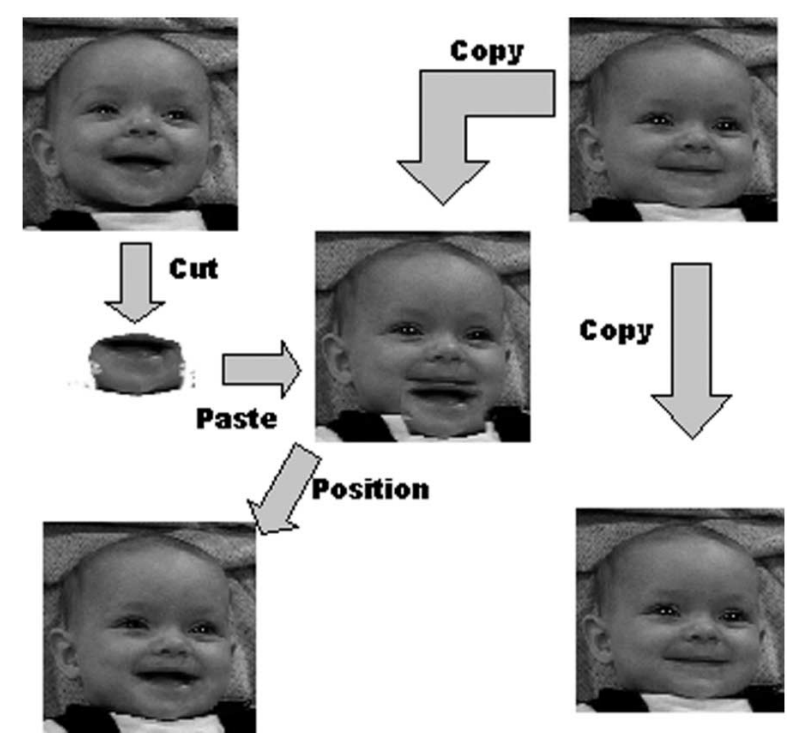

FIGURE 2 Creating a mouth opening comparison. Utilizing a base expression in which an eye constriction comparison has already been created, a second expression involving identical lateral lip corner movement but a different degree of mouth opening is identified. The mouth portion of one of these expressions (from the center of the upper lip, laterally to the lip corners, and down to the border of the face) is cut and pasted over the mouth portion of the other expression. This allows for a comparison of ratings of expressions that vary only in mouth opening.

After creating digital bases varying in eye constriction, computer vision measurements (AFA-3) were used to identify pairs of expressions with matching degrees of mouth opening or matching degrees of lip corner movement. These matched expressions were then used to digitally edit expressions in which one of these features was contrasted and the other controlled. To examine the impact of lip movement strength while controlling for mouth opening, we used two expressions with identical mouth opening but differing degrees of lip movement. This technique enabled us to examine the effect of lip movement while controlling both for mouth opening and for all indexes of the strength of the expression contained in the midface (which remained unchanged in this procedure). To examine the impact of mouth opening while controlling for strength of lip movement, we used computer vision measurements to identify two expressions with identical degrees of lip movement but varying degrees of mouth opening. The open mouth of an image was cut, pasted, and positioned over the mouth of the image with the same strength of lip movement but a lesser degree of mouth opening (see Figure 2). Because cry faces varied in degree of forehead movement, we pasted an identical forehead portion of an expression (from the eyebrows up) over all the cry face expressions of a given infant. 
Infant 4 contributed two digitally edited smiles that varied only by eye constriction produced by cheek raising (AU6). Infant 4 also contributed three digitally edited cry faces with different degrees of eye constriction. Two cry faces contrasted weak and moderate cheek raising (AU6) and were the only cry faces that did not involve lid tightening (AU7). Thus Infant 4 was the only infant who contributed smiles and cry faces in which eye constriction was produced by the same facial action. Infant 4's third cry face involved stronger cheek raising and lid tightening.

\section{Procedure}

Participants were asked to rate 29 digitally edited expressions and 8 naturally occurring expressions (see Table 1 for smiles and Table 2 for cry faces). Expressions were presented to the participants in randomized order on a computer monitor using Microsoft Access. Participants were asked to rate the contentment, joy, happiness, positive feeling, anger, distress, negative emotion, calm, and arousal they perceived in each image with a computer mouse. Ratings were made on a 9-point Likert scale, from 0 (not at all) to 8 (very strong). After rating an image, participants clicked a button to proceed to the next image.

\section{Preliminary Analyses}

Preliminary correlations were conducted to examine how ratings of specific adjectives were associated. For the sample of 95 , correlations with absolute values greater than .20 and .26 were significant, respectively, at the .05 and .01 levels. Correlations of positive feeling and joy $(M=.58$, range $=.43-.67)$, positive feeling and happiness $(M=.77$, range $=.66-.87)$, and joy and happiness $(M=.71$, range $=$ .64-.79), were high across ratings of smiles. Given these associations, a positive emotion variable was constructed by calculating the mean of happiness, joy, and positive feeling ratings. This variable was thought to reflect a composite of perceptions of positive emotion that would be mirrored by a composite negative emotion term. Correlations of negative feeling and anger $(M=.46$, range $=.22-.63)$, negative feeling and distress $(M=.68$, range $=.56-.80)$, and ratings of anger and distress $(M=.39$, range $=.24-.54)$ were relatively high across rating of cry faces. Given these associations, a negative emotion variable was constructed by calculating the mean of anger, distress, and negative feeling ratings.

We expected ratings of calm and arousal to be negatively correlated. However, preliminary analysis unexpectedly revealed slightly positive correlations between calm and arousal $(M=.09$, range $=-.13-.26)$ across all expressions. We then correlated calm and arousal with the newly formed positive emotion and negative emotion terms. As expected, arousal was modestly positively correlated with both positive emotion in smiles $(M=.36$, range $=.22-.50)$ and negative emotion in cry faces $(M=.17$, range $=.01-.26)$. Calm was unexpectedly positively correlated 
with positive emotion $(M=.23$, range $=-.029-.44)$ in smiles and negatively correlated with negative emotion $(M=-.31$, range $=-.56-.091)$ in cry faces. Given this pattern of associations, calm was not evaluated in subsequent analyses. Contentment was also not analyzed. Contentment had been chosen to index positive affect involving lower arousal levels, which is not the focus of the current report. ${ }^{1}$

\section{RESULTS}

After screening for gender effects, we report on differences in the ratings of pairs of naturally occurring expressions that differed simultaneously on three potential indexes of emotional intensity — degree of lip movement, mouth opening, and eye constriction. We then report on multivariate analyses of ratings of emotional intensity and arousal in digitally edited expressions in which the impact of each potential intensity index is examined separately.

Rater gender was not a topic of interest and analyses were conducted with and without rater gender. There were no instances in which gender interacted with a facial feature that showed a significant effect in the analyses conducted without the gender term. There was only one significant main effect of gender, and it occurred in analyses of the naturally occurring expressions. For this reason, analyses reported for the naturally occurring expressions incorporate a gender term; analyses reported for the digitally edited expressions do not.

\section{Naturally Occurring Expressions}

Smiles with stronger lip corner pulling, mouth opening, and eye constriction were rated as expressing stronger positive emotion than smiles with weaker lip pulling, minimal or no mouth opening, and no eye constriction (see Table 3). Similarly, cry faces with stronger lip stretching, mouth opening, and eye constriction were rated as expressing stronger negative emotion than cry faces with weaker lip pulling, minimal mouth opening, and minimal eye constriction. There was a main effect of gender in which female raters $(M=6.1)$ perceived Infant 3's naturally occurring cry faces as more negative than male raters $(M=5.6), F(1,93)=6.66, p=.011, \eta_{\mathrm{p}}{ }^{2}$ $=.067$, but there were no interactions between facial feature intensity and gender.

\section{Digitally Edited Expressions}

Analyses. Four of the digitally edited expression sets consisted of six expressions. Half of the expressions involved stronger eye constriction, and half involved weaker or no eye constriction. Because not all possible combinations of lip movement and mouth opening were employed, a combined factor with three levels was

\footnotetext{
${ }^{1}$ Rating data for these variables can be obtained from the second author.
} 
created in which the effects of degree of lip movement and mouth opening could be examined with prespecified contrasts. Separate 3 (strength of lip raising and mouth opening) $\times 2$ (eye constriction) repeated measures analyses of variance (ANOVAs) were performed for Infants' 1 and 2 smiles and Infants' 1 and 3 cry faces. Separate repeated measures ANOVAs were performed for Infant 4's smiles and cry faces in which eye constriction was the only factor. Separate ANOVAs were used to predict positive emotion and arousal in smiles; separate ANOVAS were also used to predict negative emotion and arousal in cry faces. We hypothesized that lip movement, mouth opening, and eye constriction would all influence perceptions of positive emotion and negative emotion, but that mouth opening would be uniquely associated with ratings of arousal.

Positive emotion perceived in smiles. For both Infants 1 and 2, smiles involving stronger lip corner pulling were rated higher on positive emotion than smiles with weaker lip corner pulling (see Table 4). For Infant 2, but not Infant 1, smiles involving greater mouth opening were rated higher in positive emotion than smiles with less mouth opening. Infant 1's open-mouth smiles involved stronger lip corner pulling as assessed by human raters but not by computer vision measurements (see Table 1). The lack of an open-mouth finding for this infant assured that this discrepancy did not bias results.

Smiles for both Infants 1 and 2 (see Figure 3) involving eye constriction were rated higher on positive emotion than the same smiles without eye constriction. Ratings of positive emotion for Infant 1 showed a Lip Movement $\times$ Eye Constriction interaction, $F(1,94)=6.78, \eta^{2}=.07, p<.05$. Only in the pair of smiles with weaker lip corner pulling, was eye constriction $(M=5.31, S D=1.46)$ rated as expressing more positive emotion than no eye constriction $(M=4.73, S D=1.49)$, $t(94)=4.34, p<.001$. Although an eye constriction effect was not present in Infant 1 's smiles with stronger lip corner pulling, Infant 4's smiles were characterized by strong lip corner pulling and the smile that involved eye constriction was rated as more positive than the same smile without eye constriction (see Table 4).

Negative emotion perceived in cry faces. Infant 3 but not Infant 1's cry faces involving stronger lip stretching were rated higher in negative emotion than cry faces with weaker lip stretching (see Table 5). Both infants' cry faces involving greater mouth opening were rated higher in negative emotion than cry faces with less mouth opening. Both infants' cry faces with strong eye constriction were rated higher in negative emotion than the same cry faces with weaker eye constriction (see Figure 3).

Infant 4 showed a general eye constriction effect on ratings of negative emotion (see Table 4). Ratings of the cry face with weak eye constriction (caused by weak cheek raising) did not differ, however, from ratings of the cry face with moderate eye constriction (caused by moderate cheek raising), the only contrast in the study 


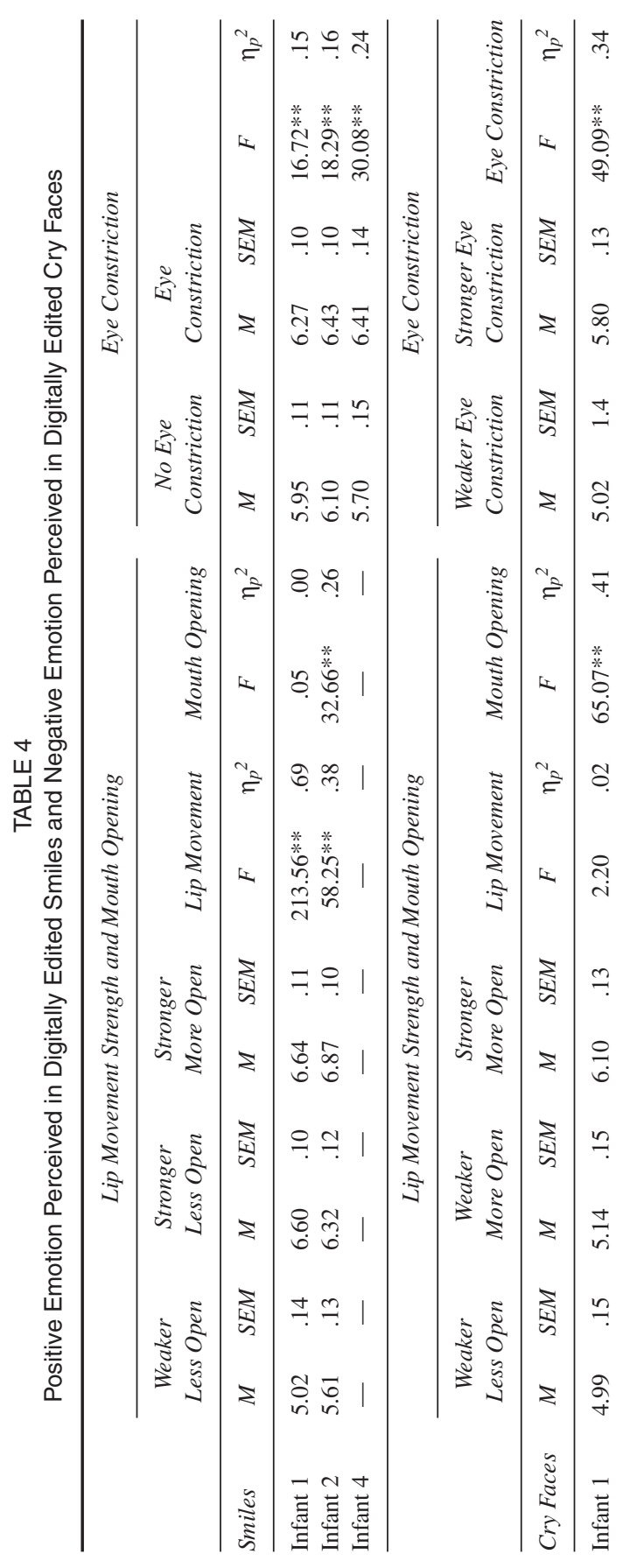




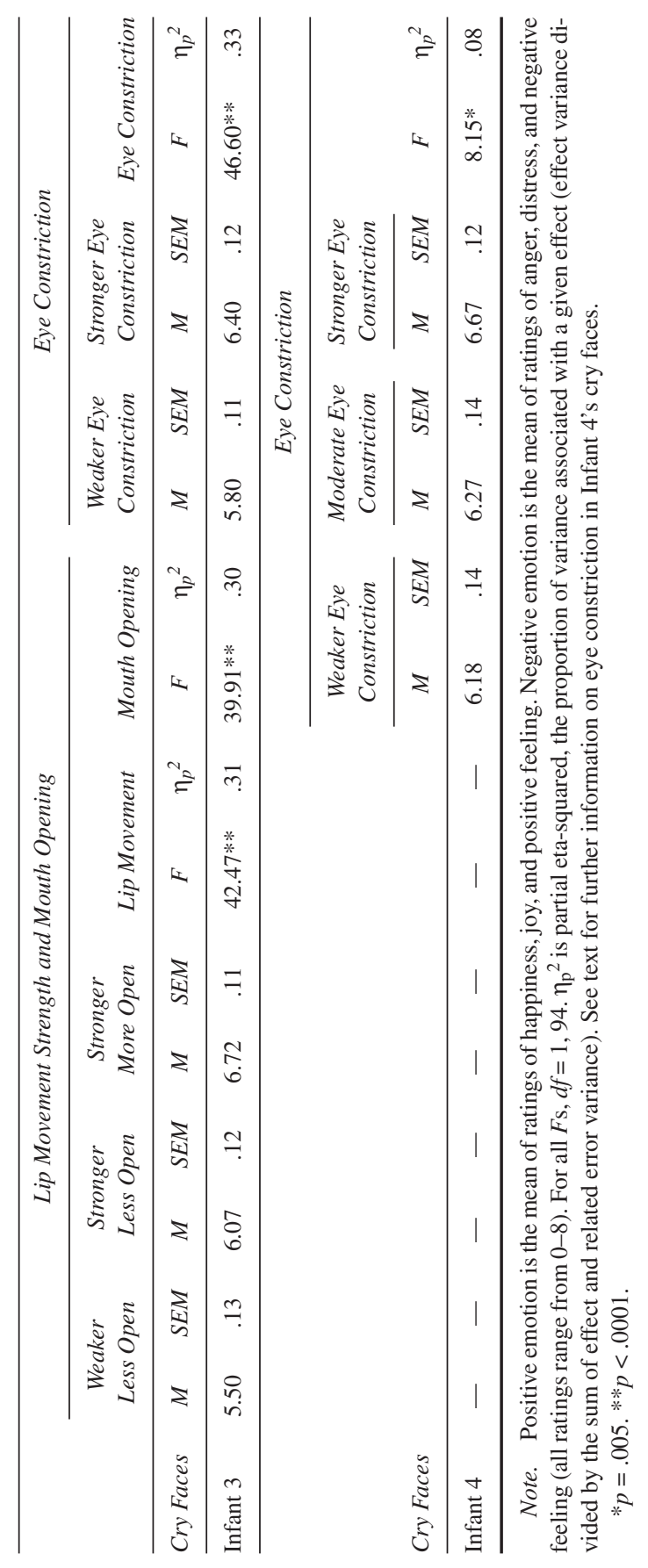




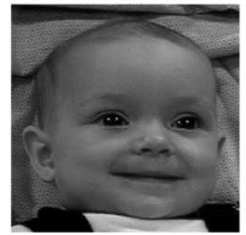

No Cheek-Raising

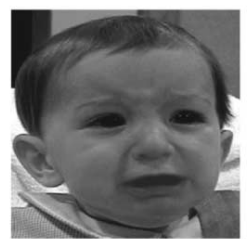

Slight Cheek-Raising

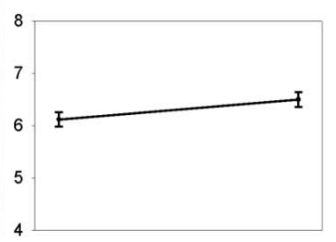

Mean ratings of Positive Emotion

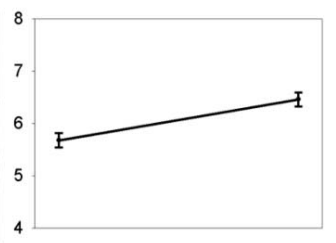

Mean ratings of Negative Emotion

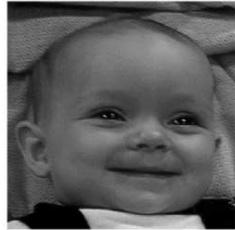

With Cheek-Raising

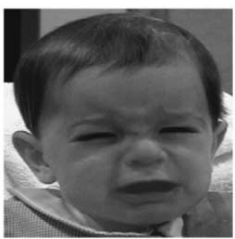

Strong Cheek-Raising

FIGURE 3 Mean ratings of positive emotion (on a scale from 0-8) and their standard errors for the pair of smiles in the Infant 2 smile set. Mean ratings of negative emotion (on a scale from $0-8$ ) and their standard errors for the pair of cry faces in the Infant 3 cry face set. In each row, identical images of the lower face have been pasted onto an image with little or no cheek raising (left) and an image with greater cheek raising (right).

in which neither cry face involved lid tightening, $F(1,94)=.470, p>.05$. The Infant 4 cry face that involved stronger eye constriction (caused by stronger cheek raising and lid tightening) was rated more negatively than the cry face involving only moderate cheek raising, $F(1,94)=9.57, \eta^{2}=.092, p<.005$, a contrast similar to that made for other infants.

Arousal perceived in smiles and cry faces. Greater lip stretching was associated with higher ratings of arousal in both infants' smiles and one of two infants' cry faces (see Table 5). Greater mouth opening was associated with higher ratings of arousal across all relevant smiles (Infants 1 and 2) and cry faces (Infants 1 and 3; see Figure 4). Individuals rated expressions with eye constriction as expressing more arousal than expressions without eye constriction in Infant 2's but not Infant 1's smiles. Infant 4's smiles with eye constriction were rated higher in arousal than her smiles without eye constriction (see Table 5). The eye constriction effect approached significance for Infant $3(p=.054)$ and Infant 1's cry faces ( $p=$ .062). Like ratings of negative emotion, ratings of arousal for Infant 1 showed a Lip Movement $\times$ Eye Constriction interaction, $F(1,94)=5.07, \eta^{2}=.051, p<.05$. Only in pairs of cry faces with weaker lip stretching, stronger eye constriction $(M=3.78$, $S D=2.04)$ was rated as expressing more arousal than weaker eye constriction $(M=$ $3.22, S D=2.20), t(94)=3.10, p<.005$. There was not an overall effect of eye constriction on arousal in Infant 4's cry faces. 


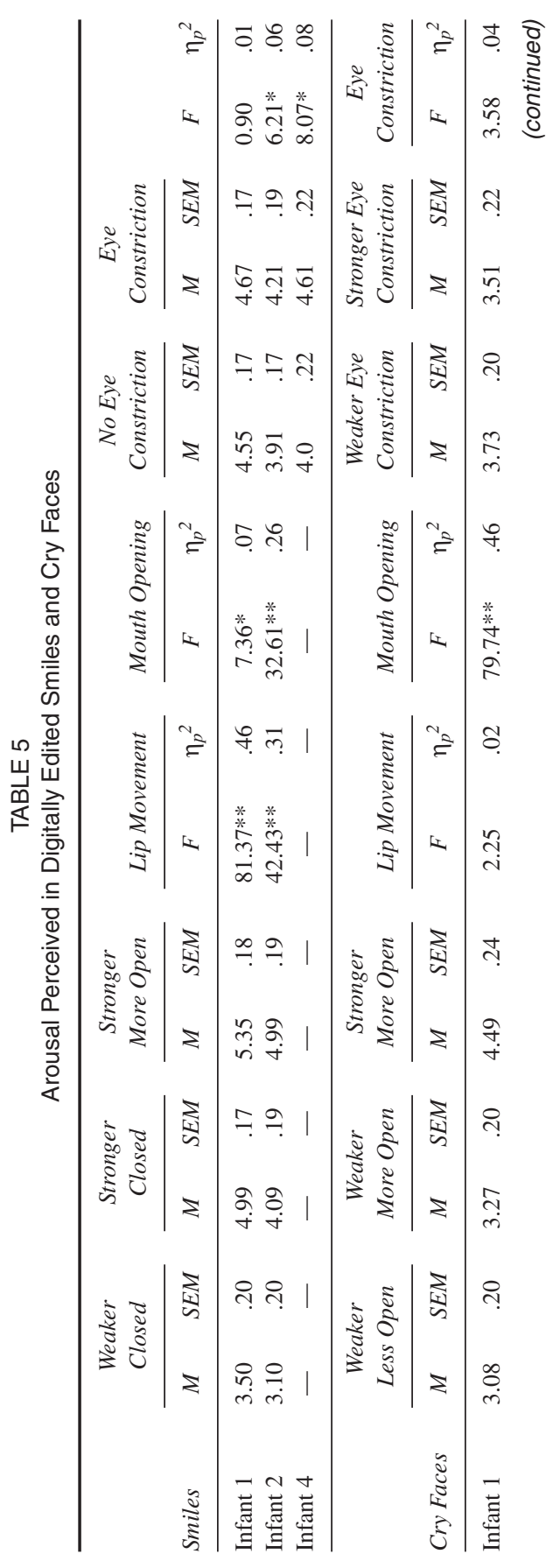




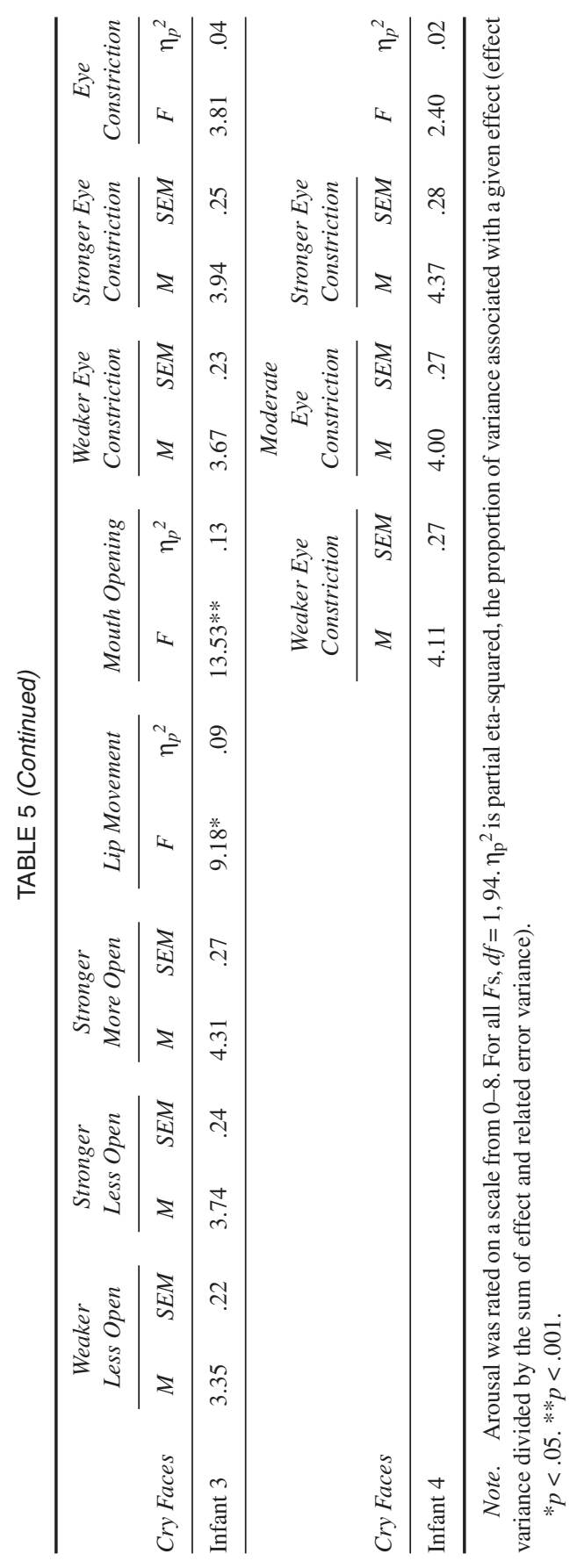




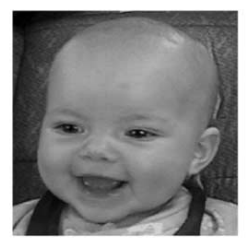

Less Mouth Opening

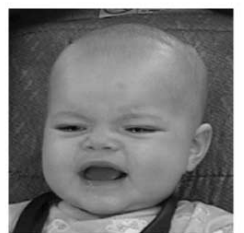

Less Mouth Opening

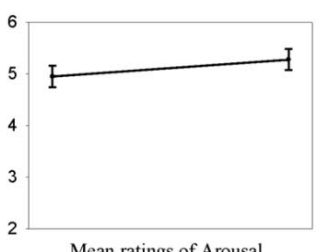

Mean ratings of Arousal

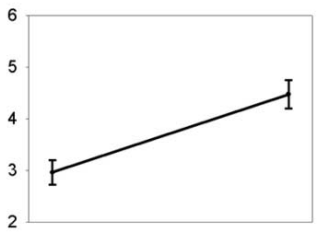

Mean ratings of Arousal

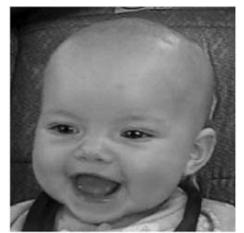

Greater Mouth Opening

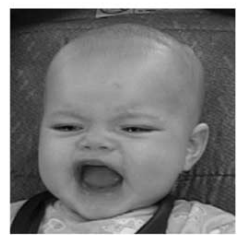

Greater Mouth Opening

FIGURE 4 Mean ratings of arousal (on a scale from 0-8) and their standard errors for the pair of smiles in the Infant 1 smile set and the cry faces in the Infant 1 cry faces set. In each row, an open mouth (right) has been pasted onto an image with less mouth opening but the same degree of lip movements (left).

\section{DISCUSSION}

Adult ratings of naturally occurring and digitally edited smiles and cry faces supported an overarching hypothesis. Similar facial movements were associated with the perception of positive emotional intensity in smiles and negative emotional intensity in cry faces, and with the perception of arousal in both types of expression. Expressions with greater eye constriction were consistently rated as expressing stronger positive emotion and stronger negative emotion than identical expressions with less or no eye constriction. Stronger lip movement was consistently associated with perceptions of greater positive emotion but not negative emotion. Stronger mouth opening was consistently associated with perceptions of greater negative but not positive emotion. Greater mouth opening, however, was associated with the perception of stronger arousal in all the digitally edited smiles and cry faces. After reviewing the study's limitations, we explore the implications of these findings for early infant emotional communication.

\section{Limitations}

Interpretation of these findings should be tempered by awareness of the study's methodological limitations. Because rated expressions were obtained from 4 infants, only effects that were consistent across all relevant infants will be interpreted. Undergraduates served as researchers in this study. Results from a separate study suggest, however, that the perceptions of college undergraduates are similar to those of infants' parents (Cassel, Messinger, Acosta, Ambadar, \& Cohn, 2005). 


\section{Perceived Positive and Negative Emotion in Naturally Occurring Expressions}

Naturally occurring infant smiles involving the co-occurrence of greater lip movement, mouth opening, and eye constriction, were perceived as expressing stronger positive emotion than smiles without these features. These very large effects are congruent with a growing literature indicating that these smiles index very high positive emotion (Dickson, Walker, \& Fogel, 1997; Fogel, Nelson-Goens, Hsu, \& Shapiro, 2000; Messinger, 2002; Messinger et al., 2001), and might be a marker for resiliency and optimal developmental outcomes Very large effects also characterized the perception of naturally occurring cry faces involving the co-occurrence of these three facial features as expressing more negative emotion than expressions without. This finding has parallels in the literature (Oster, 2000; Segal et al., 1995) and might be useful for the identification of reactions of extreme pain in infants in clinical settings. To examine this parallel between positive and negative expressions, we controlled for each of the three potential indexes of emotional intensity in the digitally edited expressions.

\section{Overview of Digitally Edited Expressions}

Ratings of naturally occurring expressions have clear face validity but cannot indicate which features are responsible for perceptions of greater intensity. Yet digital editing must proceed with attention to ecological validity, the types of expressions that infants actually produce. Greater lip movement, eye constriction, and mouth opening tend to co-occur in naturally occurring infant smiles and similar factors are operative in cry faces (Messinger, Fogel, \& Dickson, 1997, 1999; Messinger et al., 2001; Oster, 2005; Segal et al., 1995). The strength of these associative tendencies is, however, variable (Fogel et al., cited in Messinger, 2002), so that the range of possible expressions is unknown (Cassel et al., 2005; Oster, 2000). Nevertheless, the use of digital editing, insofar as facial features were forced to vary individually, rather than in concert, might represent a conservative estimate of the impact of these features. Further understanding of the dynamic constraints and possibilities in infant facial expressions might depend on the implementation of computer vision measurement techniques such as those that were used in this research to guide digital editing.

Pragmatically, the creation of digitally edited expressions indistinguishable from naturally occurring expressions involved working carefully with the constellation of features present in the original expressions. This precluded carrying out all possible mouth opening and lip corner movement contrasts in both eye constriction conditions. In addition, these three facial features were chosen because they were thought to index the intensity of both positive and negative expressions (Messinger et al., 1997). Other facial features such as brow movements might well 
impact the perceived intensity of negative expressions - this was controlled for in this study_but are unlikely to play any such role in smiles (Oster, 2005).

\section{Perceptions of Digitally Edited Expressions}

In the digitally edited expressions, infant smiles with eye constriction were consistently rated as expressing stronger positive emotion than identical smiles without eye constriction. This moderate-sized effect accounted for $15 \%$ to $24 \%$ of variance in ratings related to eye constriction, somewhat less than in previous reports (Messinger, 2002). It parallels ratings of adult (Duchenne) smiles involving eye constriction, and complements findings that infant and adult smiles involving eye constriction occur in more positive situations and involve different patterns of cerebral activation than smiles without eye constriction (Fox \& Davidson, 1988; Frank, Ekman, \& Friesen, 1993). An interaction effect in ratings of one infant's smiles suggested that the tendency of eye constriction to make smiles appear more positive is not absolute, but can be tempered by the presence of other intensity indexes.

In infant cry faces, anger and distress expressions identified by the Max coding system (Izard, Dougherty, \& Hembree, 1983), are distinguished by the tightly closed eyes (lid tightening) of the distress expression (Camras, 1992). In this study, greater eye constriction in cry faces was rated as expressing stronger negative emotion, a measure that combined anger and distress, as well as negative feeling. These were large effects, accounting for approximately a third of the relevant variance. Eye constriction in cry faces is typically produced by a combination of cheek raising and lid tightening. In the only contrast of cry faces that involved cheek raising and not lid tightening, there was no independent effect of cheek raising. Eye constriction produced by cheek raising typically makes smiles appear more positive; eye constriction produced by cheek raising and lid tightening typically makes cry faces appear more negative. Thus, the impact of eye constriction in smiles and cry faces is best thought of as indexing similar but not identical facial movements.

One of two infant's smiles with greater mouth opening were perceived as expressing stronger positive emotion than smiles with less mouth opening. Cry faces with greater mouth opening were consistently perceived as expressing more negative emotion than identical cry faces involving less mouth opening, a large effect accounting for more than $30 \%$ of relevant rater variance. Greater mouth opening, then, appears to be consistently associated with the intensity of perceived negative emotion, but less consistently associated with the intensity of perceived positive emotion.

Smiles with stronger lip corner pulling were rated as expressing more positive emotion than expressions with weaker lip corner pulling in both smile sets. Significant effects of lip movement were found for Infant 3, but not Infant 1, cry faces. The nonsignificant effect of stronger lip stretching in the Infant 1 cry face set might have been due to making this contrast in the context of greater mouth opening. In 
all other expressions, lip movement differences were examined in expressions with lesser degrees of mouth opening. Nevertheless, degree of lip movement was consistently associated with the intensity of perceived positive emotion, but not consistently associated with the intensity of perceived negative emotion.

\section{Perceived Arousal}

Greater lip movement was associated with greater perceived arousal in both infants' smiles (large effects, accounting for approximately $30 \%$ of relevant variance) but only one infant's cry faces. Eye constriction was associated with arousal in two of three infants' smiles and in only one of three infants' cry faces. In positive and negative expressions, greater mouth opening was consistently perceived as expressing stronger arousal than expressions with less mouth opening (effects accounted for $7 \%-46 \%$ of relevant variance). This finding is consonant with research from human and nonhuman primate infants on the circumstances in which open-mouth expressions occur. It suggests the hypothesis that both smiles and cry faces involving greater mouth opening should involve increases in physiological indexes of arousal (i.e., respiration and heart rate) in comparison to the same expressions involving no or lesser degrees of mouth opening.

\section{Theoretical Implications}

With the exception of research utilizing BabyFACS, basic and differential emotion perspectives have focused on canonical expressions of basic emotions, rather than investigating the range of smiles and the range of cry faces examined in this study. Basic and differential emotion theories hold that distinct affect programs control facial expressions of positive and negative emotion. From this perspective, there is no theoretical reason to suspect that distinct affect programs would use common markers of expressive intensity. Specifically, there is no a priori reason for these perspectives to predict that similar facial movements would index the increased intensity in two types of expressions thought to reflect qualitatively different emotions.

Dimensional accounts suggest that emotions differ on arousal and hedonic dimensions and that discrete emotions can be located in an emotion space composed of those dimensions. We found support for the hypothesis proposed by dimensional theorists (Russell, 1997; Smith \& Scott, 1997) and others (Bolzani et al., 2002; Messinger, 2002) that mouth opening communicates arousal across different types of emotion expression. Dimension accounts suggest, more generally, that a given facial action might have a particular meaning across a range of expressive context. However, dimensional accounts do not appear to speak to the possibility that a given facial feature (i.e., eye constriction) would make smiles appear more positive and cry faces more negative (Feldman-Barrett \& Russell, 1999). 
Theoretical work from a dynamic systems perspective originally suggested the hypothesis that some (but not all) facial movements might be associated with greater positive emotional intensity when they occur with smiles and greater negative emotional intensity when they occur with cry faces (Messinger et al., 1997). In this view, the facial constituents of an expression assemble to create emotional meaning, but the expression's meaning is greater than the sum of its constituents. Eye constriction, for example, although it has somewhat different sources in smiles and cry faces, appears to make positive expressions appear more positive and the cry face negative expression appear more negative in a multiplicative fashion. One possibility is that eye constriction communicates that one is reducing one's visual field as one is immersed in an emotional experience (Messinger, 2002). Smiles with eye constriction might convey that the smiler is only engaged with the positive experience and can see nothing else. Cry faces with greater eye constriction might convey being overwhelmed by distress so great that the expresser must plead for help.

Having common facial mechanisms for increasing the signal value of positive and negative expressions is similar to increasing the volume of a vocalization. It allows messages of different affective valences to be communicated more forcefully using a common expressive mechanism. The potential facial mechanisms identified here-lateral mouth movement, opening of the oral orifice, and constriction of the ocular orifice-might have evolved because of their perceptual salience. This view is consistent with recent evidence on the importance of bottom-up processing of emotional expressions (Dailey, Cottrell, Padgett, \& Adolphs, 2002).

\section{CONCLUSION}

Positive emotional expressions communicate and elicit a receptivity to pleasurable engagement necessary for normative infant development. Negative emotion expressions communicate unwanted experiences and the need for help from a caregiver. This research has identified facial features of smiles and cry faces that communicate increased emotional intensity. Further insight into the meaning of these expressions and their developmental consequences will require attention to the patterning of these expressions in time, their relation to other expressive behaviors such as vocalizations, research on the situations in which infants produce these expressions, as well as continued research on how the expressions are perceived.

\section{ACKNOWLEDGMENTS}

This research was supported by a General Research Support Award from the University of Miami, National Institute of Child Health and Human Development 
Grant HD41619, a grant from the Positive Psychology Network, National Science Foundation Grant 0418400, and National Institute of Mental Health Grant R01 MH51435. We gratefully acknowledge Harriet Oster for her assistance with BabyFACS and for her review of the manuscript.

\section{REFERENCES}

Bennett, D. S., Bendersky, M., \& Lewis, M. (2002). Facial expressivity at 4 months: A context by expression analysis. Infancy, 3, 97-113.

Bolzani, L., Messinger, D. S., Yale, M. E., \& Dondi, M. (2002). Smiling in infancy. In M. Abel (Ed.), An empirical reflection on the smile (pp. 111-135). New York: Edwin Mellen.

Camras, L. A. (1992). Expressive development and basic emotion. Cognition and Emotion, 6, 269-283.

Camras, L. A., Oster, H., Campos, J. J., Miyake, K., \& Bradshaw, D. (1992). Japanese and American infants' responses to arm restraint. Developmental Psychology, 28, 578-583.

Cassel, T., Messinger, D., Acosta, S., Ambadar, Z., \& Cohn, J. (2005). Production and perception of early joy: Computer measurements and human ratings of infant smiles. Manuscript in preparation.

Cohn, J. F., Campbell, S. B., \& Ross, S. (1991). Infant response in the still-face paradigm at 6 months predicts avoidant and secure attachment at 12 months. Development and Psychopathology, 3, 367-376.

Cohn, J. F., \& Kanade, T. (in press). Automated facial image analysis for measurement of emotion expression. In J. A. C. J. B. Allen (Ed.), The handbook of emotion elicitation and assessment. New York: Oxford University Press.

Cohn, J. F., Reed, L., Moriyama, T., Xiao, J., Schmidt, K., \& Ambadar, Z. (2004). Multimodal coordination of facial action, head rotation, and eye motion. In Institute of Electrical and Electronics Engineers International Conference on Automatic Face and Gesture Recognition, FG '04, Seoul, Korea. ( pp. 129-135).

Dailey, M. N., Cottrell, G. W., Padgett, C., \& Adolphs, R. (2002). EMPATH: A neural network that categorizes facial expressions. Journal of Cognitive Neuroscience, 14, 1158-1173.

Davidson, R. J., \& Fox, N. A. (1989). Frontal brain asymmetry predicts infants' response to maternal separation. Journal of Abnormal Psychology, 98, 127-131.

Dickson, K. L., Walker, H., \& Fogel, A. (1997). The relationship between smile-type and play-type during parent-infant play. Developmental Psychology, 33, 925-933.

Ekman, P., Davidson, R. J., \& Friesen, W. V. (1990). The Duchenne Smile: Emotional expression and brain physiology: II. Journal of Personality and Social Psychology, 58, 342-353.

Ekman, P., \& Friesen, W. V. (1978). Facial action coding system. Palo Alto, CA: Consulting Psychologists Press.

Feldman-Barrett, L., \& Russell, J. A. (1999). The structure of current affect: Controversies and emerging consensus. Current Directions in Psychological Science, 8, 10-14.

Fogel, A., Nelson-Goens, G. C., Hsu, H., \& Shapiro, A. F. (2000). Do different infant smiles reflect different emotions? Social Development, 9, 497-522.

Fogel, A., Hsu, H., Shapiro, A. F., Nelson-Goens, C., \& Secrist, C. (in press). Effects of normal and preturbed social play on the duration and intensity of different types of infant smiles. Developmental Psychology.

Fox, N., \& Davidson, R. J. (1988). Patterns of brain electrical activity during facial signs of emotion in 10 month old infants. Developmental Psychology, 24, 230-236.

Fox, N. A., Henderson, H. A., Rubin, K. H., Calkins, S. D., \& Schmidt, L. A. (2001). Continuity and discontinuity of behavioral inhibition and exuberance: Psychophysiological and behavioral influences across the first four years of life. Child Development, 72, 1-21. 
Frank, M. G., Ekman, P., \& Friesen, W. V. (1993). Behavioral markers and the recognizability of the smile of enjoyment. Journal of Personality and Social Psychology, 64, 83-93.

Frijida, N. H., Kuipers, P., \& Schure, E. (1989). Relations among emotion, appraisal, and emotional action readiness. Journal of Personality and Social Psychology, 57, 212-228.

Izard, C. E. (1979). The Maximally Discriminative Facial Coding System. Newark: University of Delaware.

Izard, C. E., Dougherty, L. M., \& Hembree, E. A. (1983). A system for identifying affect expressions by holistic judgements. Newark: University of Delaware, Instructional Research Center.

Kagan, J. (1997). Temperament and the reactions to unfamiliarity. Child Development, 68, 139-143.

Messinger, D. S. (2002). Positive and negative: Infant facial expressions and emotions. Current Directions in Psychological Science, 11, 1-6.

Messinger, D., Fogel, A., \& Dickson, K. L. (1997). A dynamic systems approach to infant facial action. In J. A. Russell \& F. M. Dols (Eds.), The psychology of facial expression (pp. 205-226). New York: Cambridge University Press.

Messinger, D. S., Fogel, A., \& Dickson, K. L. (1999). What's in a smile? Developmental Psychology, 35, 701-708.

Messinger, D. S., Fogel, A., \& Dickson, K. L. (2001). All smiles are positive, but some smiles are more positive than others. Developmental Psychology, 37, 642-653.

Moore, G. A., Cohn, J. F., \& Campbell, S. B. (2001). Infant affective responses to mother's still face at 6 months differentially predict externalizing and internalizing behaviors at 18 months. Developmental Psychology, 37, 706-714.

Oster, H. (2000). Baby FACS: Facial Action Coding System for Infants and Young Children. Unpublished monograph and coding manual. New York University.

Oster, H. (2003). Emotion in the infant's face: Insights from the study of infants with facial anomalies. Annals of the New York Academy of Sciences, 1000, 197-204.

Oster, H. (2005). The repertoire of infant facial expressions: An ontogenetic perspective. In J. Nadel \& D. Muir (Eds.), Emotional development (pp. 261-292). New York: Oxford University Press.

Oster, H., Hegley, D., \& Nagel, L. (1992). Adult judgments and fine-grained analysis of infant facial expressions: Testing the validity of a priori coding formulas. Developmental Psychology, 28, $1115-1131$.

Redican, W. K. (1975). Facial expressions in nonhuman primates. In L. A. Rosenblum (Ed.), Primate behavior: Developments in field and laboratory research (Vol. 4, pp. 103-194). New York: Academic.

Russell, J. A. (1997). Reading emotions from and into faces: Resurrecting a dimensional-contextual perspective. In J. A. Russell \& F. M. Dols (Eds.), The psychology of facial expression (pp. 295-320). New York: Cambridge University Press.

Segal, L., Oster, H., Cohen, M., Caspi, B., Myers, M., \& Brown, D. (1995). Smiling and fussing in seven-month-old preterm and full-term Black infants in the still-face situation. Child Development, 66, 1829-1843.

Smith, C., \& Scott, H. S. (1997). A Componential Approach to the meaning of facial expressions. In J. A. Russell \& F. M. Dols (Eds.), The psychology of facial expression (pp. 229-254). New York: Cambridge University Press.

Sroufe, A. (1979). Socioemotional development. In J. Osofsky (Ed.), Handbook of infant development (pp. 462-516). New York: Wiley.

Tronick, E., Als, H., Adamson, L., Wise, S., \& Brazelton, B. (1978). The infant's response to entrapment between contradictory messages in face-to-face interaction. American Academy of Child Psychiatry, 17, 1-13.

Xiao, J., Kanade, T., \& Cohn, J. F. (2003). Robust full motion recovery of head by dynamics templates and re-registration techniques. International Journal of Imaging Systems and Technology, 13, B85-B94. 